\title{
Brain Signaling in Psychiatric Disorders: What Can They Tell Us in the Absence of Behavioral Differences?
}

\author{
Jodi M. Gilman1,2, James M. Bjork3 ${ }^{3}$ Timothy E. Wilens ${ }^{2,4}$ \\ ${ }^{1}$ Center for Addiction Medicine, Massachusetts General Hospital, Boston, MA, USA \\ ${ }^{2}$ Department of Psychiatry, Harvard Medical School, Boston, MA, USA \\ ${ }^{3}$ Department of Psychiatry, Virginia Commonwealth University, Richmond, VA, USA \\ ${ }^{4}$ Clinical and Research Programs in Pediatric Psychopharmacology, Adult ADHD Massachusetts General \\ Hospital, Boston, MA, USA \\ Email: jgilman1@partners.org
}

Received 10 June 2015; accepted 25 July 2015; published 28 July 2015

Copyright (C) 2015 by authors and Scientific Research Publishing Inc.

This work is licensed under the Creative Commons Attribution International License (CC BY). http://creativecommons.org/licenses/by/4.0/

(c) (i) 0pen Access

\begin{abstract}
This is a commentary on the often-observed phenomenon of observing task-based brain signaling differences between clinical populations and healthy control participants in the absence of any behavioral decrements in the clinical group. We offer several explanations for why brain-based differences amid normative performance may be of interest to researchers and clinicians. First, neural processing in the clinical group may not be as efficient as that in the control group. Second, differences in activation could reveal important differences in the cognition behind the (normative) behavior. Third, differences in activation may be prognostic biomarkers of injury or decline. In addition, we contend that similar behavior between groups is important in properly interpreting brain data. Finally, we offer caveats and future directions to further clarify brain mechanisms underlying behavior in clinical populations.
\end{abstract}

\section{Keywords}

fMRI, Neuroimaging, Normative Behavior, Normative Performance, Neural Efficiency, Prognostic Biomarker

\section{Introduction}

Functional magnetic resonance imaging (fMRI) has exploded in the past decade, allowing us to observe in real time areas of the brain that are active when people perform a task, such as processing information, making deci- 
sions, or other types of behavior. Task-based fMRI experiments allow researchers to create colorized brain maps that show regions that are "activated" by a particular task. For instance, when participants are shown pictures of angry or fearful faces, brain regions that are involved in emotional processing, such as the amygdala, may be activated. Researchers have used fMRI to compare patient and control groups in order to better understand the neural mechanisms underlying specific psychiatric disorders and/or behavioral clusters of symptoms: a classic being amygdala reactivity to threatening stimuli in manic-bipolar groups. Yet, at times, differences in neural activation emerge in an affected group even when behavior on a specific task does not differ relative to controls. For example, patients and controls may have the same number of errors during a task; however, the patients may show heightened activation in brain regions involved in error-monitoring. In this article, we briefly explore the potential meaning of covert brain signatures in the apparent absence of behavioral abnormalities in a clinical group. We contend that although behavior may not differ between groups, the thoughts and processing underlying these behaviors (reflected in neural activation) may uncover unique abnormalities in the clinical sample.

\section{Different Patterns of Brain Activation amid Suboptimal Performance}

To test whether the brains of people with certain disorders function differently, researchers typically recruit a patient and a control group, and test for group differences in behavior and in correlated neural activation. In some reports, the brains of the patient group are "caught in the act" of performing suboptimally. In many cases, the behavioral deficits are then related to brain activation. Measures such as task accuracy, reaction time, or decisions can correlate directly with activation in a mechanistically-relevant brain region. For instance, in a study using an inhibitory control go-no/go task, researchers found that ADHD children had lower accuracy scores than controls, and also showed reductions in cerebellar activity in the ADHD children [1]. Such a finding allows researchers to conclude that an area of the brain (in this case, the cerebellum) is important for a particular function, and when that area of the brain is not working efficiently, behavioral performance suffers.

\section{Different Patterns of Brain Activation amid Normative Performance}

In other cases, no differences in behavioral performance between two groups are observed despite markedly different patterns of brain activation. As an example, adolescent marijuana users do not differ from non-users on behavioral tests of inhibitory control; however, marijuana users show greater neural activation than non-users during inhibition trials in right dorsolateral prefrontal cortex, a region important in inhibitory control, as well as in other brain regions [2]. Or consider an emotional discrimination task, in which individuals with schizophrenia and healthy controls do not differ on the ability to identify facial emotions, but relative to controls, the individuals with schizophrenia fail to activate the amygdala or hippocampus-regions underlying emotional processingwhile completing the same task in the scanner [3]. The interpretation of these findings becomes a bit more complex.

Why might this be? One possibility is that our "in vitro" tasks may not generalize "in vivo". In other words, researchers design tasks to capture behavioral manifestations of disorders, but the artificial MRI environment renders it difficult to truly create experimental psychology tasks that capture real-world behaviors. The absence of behavioral decrements in a clinical group may occur in tasks that are either insufficiently difficult, or that insufficiently evoke the behavior that is ostensibly awry in the presentation of the disorder [4]. Many neuropsychological tasks were developed for severely ill individuals (e.g. those with traumatic brain injury or dementia), and have later been applied to other less severe disorders that may have more subtle behavioral manifestations. Another complicating factor is that participants are likely in a state of unusual vigilance when tested in an unnatural and intimidating fMRI environment under observation. For example, compared to non affected controls, a child with ADHD may find it more difficult to sit through a lecture, or an adolescent binge drinker may take risks such as getting in a car with someone who has been drinking. We may use standard go-no/go tasks, or reward/risk-taking tasks that attempt to capture impaired attention, or increased risk-taking, but we may find no behavioral decrement emerging during the actual scan. In these cases of similar behavior, differences in neural activation may provide clues as to the behavioral decrements observed in real-world environments where vigilance is not artificially elevated.

The value of conducting expensive fMRI scans instead of simply conducting behavioral tasks lies in this ability to interpret different brain signatures underlying behavior. Functional MRI measures complex parameters such as the magnitude of activation (how strongly the region is activating), the locations of activation, and how 
these activations can change across the lifespan, during disease and recovery, or with acute pharmacological intervention. These brain signatures add a component of richness that cannot always be captured by measuring behavior alone.

\section{The "Efficiency" Account of Brain Activation during Cognitive Performance}

Imagine if your old car can still drive 60 miles in 60 minutes, but now requires $10 \mathrm{~L}$ of gasoline to do so where it once only required $7 \mathrm{~L}$. Would this indicate a problem with the engine? In numerous fMRI studies that find greater activation in terms of intensity (i.e. how strongly a brain area activates) or spatial extent (i.e. how large the area of the brain activation extends) when the patient is performing at the level of controls, it can be argued that his or her brain is performing in a less efficient manner. This "less is more" interpretation (of cortical activation in particular) is supported by findings that greater activation itself implies a harder-working brain. For example, as working memory demand increases, frontocortical activation increases [5] [6]. Conversely, as people get better at a task, their brain activations can lessen [7], suggesting that a well-practiced brain can perform the task with greater ease. Increased activation amid normal performance in certain groups can thus be interpreted as pathologically-based inefficiency, or perhaps as evidence of a brain working harder to achieve the same ends. Sullivan and Pfefferbaum refer to this phenomenon as a "process-demanding compensatory shift", and suggest that this compensation limits the brain's efficiency and capacity to process information [8]. This inefficiency could be problematic in more complex real-world situations where decisions become more complex and attention is not artificially stoked.

This "inefficiency" interpretation is widespread in studies of working memory in schizophrenic patients, and in developmental comparisons between children and adults. Schizophrenic patients typically show greater frontocortical activation during working memory than controls, even when the task difficulty is titrated to normalize performance between patients and controls [9]. Another example of this increase in magnitude comes from the Alzheimer's literature, where researchers found a greater activation in brain regions necessary for tasks requiring memory among the carriers of an Alzheimer's risk allele than among non-carriers [10]. These results suggest that persons at risk for Alzheimer's perform additional cognitive work to bring memory-related performance to a normal level. The field of alcoholism is also rich in literature showing differences in neural responses between alcohol-dependent patients and controls despite intact behavior in cognitive processes such as working memory [11] [12], emotional processing [13], and risk-taking [14]. Reduced efficiency of the frontal cortex has also been inferred by greater spatial extent of activated voxels, where normal development features a shift from a more expansive and diffuse pattern of frontocortical recruitment by cognitive tasks in childhood to a more focal but more strongly activated region in adulthood [15].

\section{Differences in Activation Could Reveal Important Differences in the Cognition behind the (Normative) Behavior}

Some studies report that a clinical group uses brain regions that are not used by the control group, despite normative task performance. In these cases, it is important to keep in mind that overt behavior is the endpoint of a cascade of neural processes that drive the behavior. Though behavioral output may be the same, the thoughts and processing underlying these behaviors may differ in the clinical sample.

For instance, in a study examining accrual of risky rewards in SUD, patients showed reduced activation in conflict-processing region of the anterior cingulate cortex, yet took no greater number of risks than controls [16]. This functional difference could be considered clinically meaningful, if, for example, the patient group made decisions based on a simple rule-based heuristic ("I'll let myself go to 60 cents every trial no matter what"), while controls experienced cognitive conflict and behavioral monitoring ("Is it worth the risk? How much am I willing to lose? How have I been doing on previous trials?”), and may have altered strategy across trials in order to optimize behavior. This would crudely suggest that SUD patients don't fully engage in the task in the same way as controls. Similarly, behaviorally inhibited children (defined by heightened vigilance, negative effect, and reactivity to novelty compared with non-inhibited peers) showed greater activation of a reward region of the brain while anticipating monetary gain or loss compared to non-inhibited peers, despite similar reaction times to win money [17]. Increased activation by reward cues could be a signature of hypervigilance characteristic of anxiety spectrum disorders. Task success in inhibited adolescents could have been driven more by fear of failure than by the excitement of gaining rewards. These differences cannot always be teased apart by examining the 
behavior in the task, and may not even be evident in self-report measures, but activation of brain regions may provide insight into these possible differences.

\section{Differences in Activation May Be Prognostic Biomarkers of Injury or Decline}

The medical field already understands the importance of assessing biomarkers in the absence of clinical symptoms; it is understood that early markers may predict later "disease states". For instance, graded exercise testing may elicit subtle cardiac disease that left untreated, may not manifest for many years. Research is emerging in neuroimaging showing that activation differences might be a covert marker or early detection marker of impairment. For example, high school football players who had suffered head collisions exhibited no clinicallyobserved symptoms associated with concussion compared to other players, but recruited different areas of the brain to complete a working memory task. There was a also a direct correlation between the amount the brain recruited other areas to complete tasks and the number of "hits" sustained during play (via helmet sensors) [18]. These data suggest that fMRI may be more sensitive than overt behavior to detect injury. fMRI can also identify covert makers of decline. In a study of Alzheimer's patients, increased baseline activation significantly correlated with the degree of decline in verbal recall two years later [10]. These and other studies suggest that observing compensatory or alternative activations in neural circuitry, even with intact behavioral performance, may be a covert or advanced marker of vulnerability that may precede overt performance deterioration.

\section{Similar Behavior between Groups Helps Us to Properly Interpret Brain Data}

Finally, we note that similar behavior or task success between groups lends its own clarity to interpretation of brain signal differences. When measuring behavioral responses, it is difficult to determine whether a specific behavior is cleanly isolated. In clinical groups, a behavioral response can be an amalgam of traits such as frustration and anger that are linked to the performance of the task. For example, when testing children with ADHD on an impulse control task, a measured behavior of "impulse control" can really be a measure of impulse control plus decay in vigilance plus frustration. Having similar task outcome normalizes the playing field and allows a better understanding of underlying "clean neurocircuitry" without the confounds of differing behavioral/task outcomes.

Furthermore, there are statistical and methodological advantages to normalizing behavior between groups. More occurrences of error feedback in one group versus another could yield spurious inferences of greater activation in the error-prone group. This has been referred to as the "Task B Problem" [19] and presents challenges when interpreting cross-sectional differences between clinical or age groups that differ markedly in cognitive functioning. Equal performance between groups avoids such confounds.

\section{Caveats and Future Directions}

Interpretation of fMRI activation remains deeply speculative, where an investigator can concoct a seemingly-sensible account of either increased or decreased activation that fits with broader clinical impressions of an age group, clinical condition, or a set of symptoms. Further research is needed for the field to develop a greater consensus understanding of "when less is more". Extensive longitudinal datasets in which participants are scanned before the onset of the disorder, during the course of the disorder, and after treatment may also provide more specificity to predicting risk for, and outcome of, psychiatric disorders and/or symptom clusters. Such datasets will allow us to form clearer interpretations of neurological differences between groups while adding to the critical literature on the prevention of psychiatric illness.

In the meantime, we contend that research findings, which communicate neural differences in the locations or magnitudes of activation in the absence of behavioral differences, should not be reflexively dismissed as clinically irrelevant, as these findings will help us to gain a better understanding of how the disordered brain (still) functions.

\section{References}

[1] Durston, S., Davidson, M.C., Mulder, M.J., et al. (2007) Neural and Behavioral Correlates of Expectancy Violations in Attention-Deficit Hyperactivity Disorder. Journal of Child Psychology and Psychiatry, and Allied Disciplines, 48, 881889. http://dx.doi.org/10.1111/j.1469-7610.2007.01754.x 
[2] Tapert, S.F., Schweinsburg, A.D., Drummond, S.P., et al. (2007) Functional MRI of Inhibitory Processing in Abstinent Adolescent Marijuana Users. Psychopharmacology, 194, 173-183. http://dx.doi.org/10.1007/s00213-007-0823-y

[3] Gur, R.E., McGrath, C., Chan, R.M., et al. (2002) An fMRI Study of Facial Emotion Processing in Patients with Schizophrenia. The American Journal of Psychiatry, 159, 1992-1999. http://dx.doi.org/10.1176/appi.ajp.159.12.1992

[4] Casey, B.J. and Durston, S. (2006) From Behavior to Cognition to the Brain and Back: What Have We Learned from Functional Imaging Studies of Attention Deficit Hyperactivity Disorder? The American Journal of Psychiatry, 163, 957-960. http://dx.doi.org/10.1176/appi.ajp.163.6.957

[5] Kirschen, M.P., Chen, S.H., Schraedley-Desmond, P. and Desmond, J.E. (2005) Load- and Practice-Dependent Increases in Cerebro-Cerebellar Activation in Verbal Working Memory: An fMRI Study. Neuroimage, 24, 462-472. http://dx.doi.org/10.1016/j.neuroimage.2004.08.036

[6] Seidman, L.J., Breiter, H.C., Goodman, J.M., et al. (1998) A Functional Magnetic Resonance Imaging Study of Auditory Vigilance with Low and High Information Processing Demands. Neuropsychology, 12, 505-518. http://dx.doi.org/10.1037/0894-4105.12.4.505

[7] Chen, Z., Lei, X., Ding, C., Li, H. and Chen, A. (2012) The Neural Mechanisms of Semantic and Response Conflicts: An fMRI Study of Practice-Related Effects in the Stroop Task. Neuroimage, 66C, 577-584.

[8] Sullivan, E.V. and Pfefferbaum, A. (2005) Neurocircuitry in Alcoholism: A Substrate of Disruption and Repair. Psychopharmacology, 180, 583-594. http://dx.doi.org/10.1007/s00213-005-2267-6

[9] Tan, H.Y., Sust, S., Buckholtz, J.W., et al. (2006) Dysfunctional Prefrontal Regional Specialization and Compensation in Schizophrenia. The American Journal of Psychiatry, 163, 1969-1977. http://dx.doi.org/10.1176/ajp.2006.163.11.1969

[10] Bookheimer, S.Y., Strojwas, M.H., Cohen, M.S., et al. (2000) Patterns of Brain Activation in People at Risk for Alzheimer's Disease. The New England Journal of Medicine, 343, 450-456. http://dx.doi.org/10.1056/NEJM200008173430701

[11] Pfefferbaum, A., Desmond, J.E., Galloway, C., Menon, V., Glover, G.H. and Sullivan, E.V. (2001) Reorganization of Frontal Systems Used by Alcoholics for Spatial Working Memory: An fMRI Study. NeuroImage, 14, 7-20. http://dx.doi.org/10.1006/nimg.2001.0785

[12] Desmond, J.E., Chen, S.H., DeRosa, E., Pryor, M.R., Pfefferbaum, A. and Sullivan, E.V. (2003) Increased Frontocerebellar Activation in Alcoholics during Verbal Working Memory: An fMRI Study. NeuroImage, 19, 1510-1520. http://dx.doi.org/10.1016/S1053-8119(03)00102-2

[13] Gilman, J.M., Davis, M.B. and Hommer, D.W. (2010) Greater Activation in Left Hemisphere Language-Related Regions during Simple Judgment Tasks among Substance-Dependent Patients in Treatment for Alcoholism. Alcoholism: Clinical and Experimental Research, 34, 331-341. http://dx.doi.org/10.1111/j.1530-0277.2009.01095.x

[14] Gilman, J., Smith, A.R., Bjork, J.M., Ramchandani, V.A., Momenan, R. and Hommer, D.W. (2015) Cumulative Gains Enhance Striatal Response to Reward Opportunities in Alcohol-Dependent Patients Addiction Biology. Addiction Biology, 20, 580-593.

[15] Durston, S., Davidson, M.C., Tottenham, N., et al. (2006) A Shift from Diffuse to Focal Cortical Activity with Development. Developmental Science, 9, 1-8. http://dx.doi.org/10.1111/j.1467-7687.2005.00454.x

[16] Bjork, J.M., Momenan, R., Smith, A.R. and Hommer, D.W. (2008) Reduced Posterior Mesofrontal Cortex Activation by Risky Rewards in Substance-Dependent Patients. Drug and Alcohol Dependence, 95, 115-128. http://dx.doi.org/10.1016/j.drugalcdep.2007.12.014

[17] Guyer, A.E., Nelson, E.E., Perez-Edgar, K., et al. (2006) Striatal Functional Alteration in Adolescents Characterized by Early Childhood Behavioral Inhibition. The Journal of Neuroscience, 26, 6399-6405. http://dx.doi.org/10.1523/JNEUROSCI.0666-06.2006

[18] Talavage, T.M., Nauman, E.A., Breedlove, E.L., et al. (2014) Functionally-Detected Cognitive Impairment in High School Football Players without Clinically-Diagnosed Concussion. Journal of Neurotrauma, 31, 327-338. http://dx.doi.org/10.1089/neu.2010.1512

[19] Church, J.A., Petersen, S.E. and Schlaggar, B.L. (2010) The "Task B Problem” and Other Considerations in Developmental Functional Neuroimaging. Human Brain Mapping, 31, 852-862. http://dx.doi.org/10.1002/hbm.21036 